01

\title{
Симметрия икосаэдра и оптические свойства фуллерена $\mathrm{C}_{60}$
}

\author{
() А.Г. Сухарев \\ Троицкий институт инновационных и термоядерных исследований, \\ 108840 Троицк, Москва, Россия \\ e-mail: sure@triniti.ru
}

Поступила в редакцию 20.07.2020 г.

В окончательной редакции 03.11.2020 г.

Принята к публикации 03.11.2020 г.

Для анализа схемы уровней фуллерена использована теория молекулярных орбиталей. Взаимодействие $\pi$ электронов, вследствие перескоков между соседними атомами углерода, приводит к образованию коллективных состояний, обладающих симметрией усеченного икосаэдра. Показано, что симметрия группы икосаэдра формирует необычный спектр уровней, связанный с видом угловой части волновых функций. Дана интерпретация неприводимых представлений группы вращений икосаэдра через сферические функции. Представлены формулы расчета сечений возбуждения электронных уровней и времени спонтанной релаксации. Показано, что данные времена спонтанной релаксации на порядки уступают временам безызлучательной релаксации, которая возникает из-за эффекта Яна-Теллера, ввиду спонтанного нарушения симметрии при взаимодействии с фононным спектром. Из-за быстрых безызлучательных процессов, несмотря на существование спектра поглощения, спектр излучения в оптической области не регистрируется. Вместо него регистрируется тепловое излучение в области частот фононного спектра. Предложены методы обхода проблемы гашения электронного спектра излучения.

Ключевые слова: группа икосаэдра, неприводимые представления, сечение возбуждения и время спонтанной релаксации перехода.

DOI: $10.21883 /$ OS.2021.02.50550.204-20

\section{1. Введение}

Наиболее интересным представителем точечных групп является группа икосаэдра. С помощью теории представлений свойства групп можно описывать с помощью операций над матрицами. В рамках теории молекулярных орбиталей Хюккеля можно свести квантовое описание молекулы фуллерена $\mathrm{C}_{60}$ к матричному представлению с рангом матрицы 60. В рамках этого описания волновая функция задается только в узлах молекулы, в которых находятся атомы углерода. В промежутках между узлами волновую функцию следует аналитически продолжить. В данном случае оказывается удобно использовать сферические функции, поскольку молекула фуллерена имеет форму почти идеального футбольного мяча. Молекула фуллерена представляет собой усеченный икосаэдр, в котором вместо 12 вершин исходного икосаэдра после усечения становиться в пять раз больше вершин. Сетка из параллелей и меридианов, проведенная через узлы молекулы, неравномерна, что приводит к интересным последствиям. Волновые функции неприводимых представлений усеченного икосаэдра могут быть разложены по сферическим функциям, коэффициенты разложения обладают зависимостью от параметров силы связи атомов углерода с соседями, а значит от длины соответствующих ребер в усеченном икосаэдре.

Первоначально интерес к икосаэдру проявляли математики из-за обнаруженной связи между геометри- ей и алгеброй. Клейн исследовал решения уравнения пятой степени с помощью симметрии икосаэдра [1]. Интерес физиков к симметрии икосаэдра разгорелся в полную силу после обнаружения в природе объектов, обладающих данной симметрией [2]. Анализ спектра электронных уровней для молекулы $\mathrm{C}_{60}$ с помощью симметрии икосаэдра дан в работе [3] в рамках теории молекулярных орбиталей. Спектр собственных значений электронных уровней молекулы расщепляется на семейства в соответствии с неприводимыми представлениями, следующими из теории групп. Метод молекулярных орбиталей Хюккеля предполагает, что основной вклад в гамильтониан вносит взаимодействие только ближайших соседей. У каждого углерода в молекуле $\mathrm{C}_{60}$ таких coceдей три. Матрица гамильтониана имеет ранг 60. С помощью симметрии вместо нее можно изучать спектр с помощью матрицы размера $6 \times 6$ [4]. Обнаруженный подход позволил найти аналитические решения как для спектра собственных значений, так и для собственных волновых функций в дискретном представлении. Оказалось, что для этого нужно решить либо квадратное, либо кубическое алгебраическое уравнение. И это несмотря на то, что исходная матрица имеет размерность 60 . Мы видим, что свойства данной симметрии позволяют делать совершенно необыкновенные вещи. И как это делать, представляет особый интерес.

На поверхности молекулы фуллерена атомы углерода формируют узор из пятиугольников и шестиугольников. Поэтому матричный элемент, определяющий энергию 
перескока электрона между соседними положениями, определяется двумя константами. Первая - это связь внутри пятиугольного кольца, вторая - параметр перехода по двойной связи, соединяющей различные пятиугольники. Ось симметрии, проходящая через центр пятиугольника (и центр молекулы) - ось пятого порядка, ось, проходящая через диаметрально противоположные шестиугольники - ось третьего порядка. Кроме того, есть операции инверсии и повороты на угол $\pi$ относительно оси, проходящей через центр общих ребер двух смежных шестиугольников. Всего группа насчитывает 120 элементов, которые разбиваются на десять классов и формируют десять типов неприводимых представлений. Для них строится таблица характеров, которая как отпечатки пальцев фиксирует их индивидуальность. Для характеров вводится скалярное произведение. Два разных неприводимых представления оказываются ортогональны. Сравнивая с таблицей характеров для орбитального момента (на введенном наборе поворотов), можно заключить, что для электронов в коллективном состоянии существуют чистые представления с орбитальным моментом от нуля до двух. Состояния электронов с более высоким орбитальным моментом разбиваются на прямую сумму нескольких представлений, которые в общем случае имеют и различную энергию уровней. После этих вводных замечаний рассмотрим более подробно теорию симметрии икосаэдра.

\section{2. Симметрия правильного усеченного икосаэдра}

Правильный икосаэдр является представителем платоновских правильных выпуклых многоугольников (рис. 1). Данное тело содержит 12 вершин, 20 граней и 30 ребер. Ориентируем одну из осей, проходящих через противоположные вершины, по оси $z$. Из верхней вершины спускаются пять лучей к пяти вершинам. Получается пятиугольная пирамида, боковые стороны которой образованы правильными треугольниками. Одна из вершин основания пирамиды пусть будет ориентирована вдоль оси $x$. Расположим тело симметрично относительно плоскости $x y$, чтобы нижняя вершина имела ту же координату, но с противоположным знаком. Тогда над плоскостью и под ней в точности по 6 точек. Разместив в вершинах икосаэдра по атому углерода, получим молекулу $\mathrm{C}_{12}$.

Если от каждой вершины правильного икосаэдра отсечь правильную пятигранную пирамиду одной и той же высоты, то получится правильный усеченный икосаэдр, который схематично изображен на рис. 2. Его симметрия совпадает с симметрией исходного тела. Однако каждая вершина икосаэдра превращается в пять: $B=12 \times 5=60$. Соответственно увеличивается число ребер - $P=30+12 \times 5=90$ и граней $\Gamma=20+12=32$. По формуле Эйлера число вершин, граней и ребер связаны формулой $\Gamma+B=P+2$. Теперь

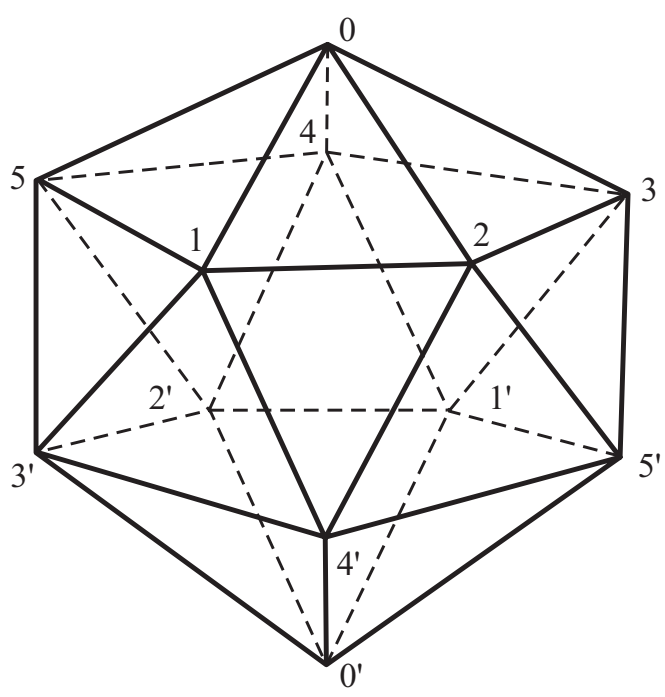

Рис. 1. Платонов правильный выпуклый икосаэдр.

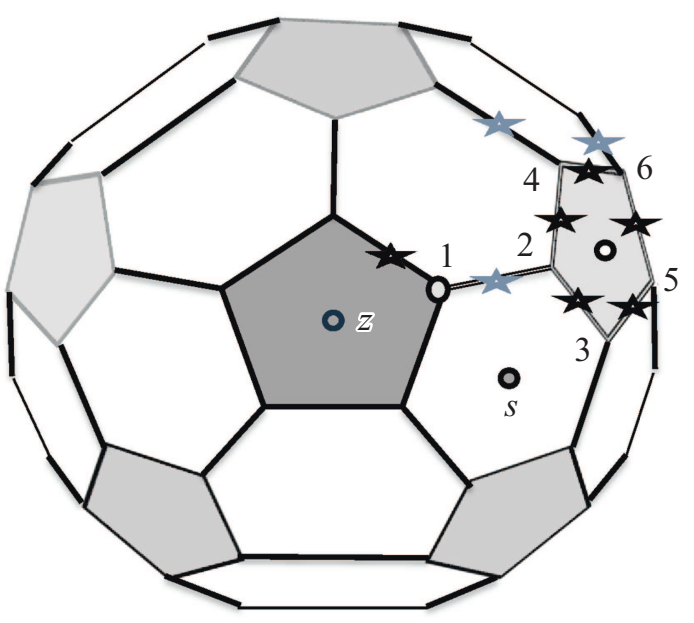

Рис. 2. Правильный усеченный икосаэдр.

мы можем отслеживать состояние волновой функции в 60 точках в рамках модели молекулярных орбиталей Хюккеля. Поскольку группа икосаэдра содержит 120 элементов, из которых 60 соответствуют поворотам, то увеличение числа вершин приводит к таблице характеров $10 \times 10$ (табл. 1$)$.

В соответствии с принятыми обозначениями синглет обозначается как $A$, триплеты как $T_{1}$ и $T_{2}$, а четырехлибо пятикратно вырожденные состояния системы как $G, H$. Индекс $g$ означает четное представление, а $u$ - нечетное относительно центральной инверсии $P$. Каждая колонка задает элемент группы преобразований в матричном представлении. Первая колонка - это единичная матрица, отвечающая в группе единичному элементу (тождественному преобразованию). Размерность матрицы преобразования такова же, как и размерность представления. Поэтому шпур матрицы (характер) 1, 3, 4, 5 соответственно. Следующие две колонки отвечают 
повороту относительно шести осей пятого порядка. По определению повороты $G_{a}$ и $G_{b}$ входят в один класс сопряженных элементов, если преобразуются друг в друга в результате всех операций $G_{x}$ группы: $G_{a}=G_{x} G_{b} G_{x}^{-1}$. Один класс сопряженных поворотов происходит на угол $2 \pi / 5$, второй - на угол $4 \pi / 5$. Число элементов класса равно 12 (равно числу вершин икосаэдра). Другой класс сопряженных поворотов соответствует повороту относительно десяти осей третьего порядка. Они проходят через центры противоположных граней. Общее количество элементов класса равно 20 по количеству граней икосаэдра. Поворотов относительно оси третьего порядка три: на углы $0, \pm 2 \pi / 3$. После вычитания тождественного преобразования остаются прямой и обратный поворот на 120 градусов. Однако поворот против часовой стрелки для противоположной относительно инверсии грани соответствует повороту по часовой стрелке для ее внешней нормали. Относительно осей второго порядка можно сказать, что их 15 и проходят они через середины противоположных ребер. Так как прямое и обратное преобразования при повороте на угол $\pi$ совпадают, то класс содержит 15 сопряженных элементов. С учетом операции инверсии таблица содержит еще пять колонок. Так, $S_{10}^{3}=C_{5} P$ и т. д. Рассмотрим колонку $12 C_{5}$. Одномерное представление содержит вновь единицу, у пятимерного неприводимого представления $H_{g}$ на диагоналях лежат числа $e^{i 2 \pi k / 5}-$ каждое отвечает собственному вектору со своим поворотом на угол, кратный $2 \pi / 5$. Их сумма равна 0 . Для трехмерного представления $T_{1 u}$ сумма элементов на диагонали содержит только три экспоненты: $e^{i 2 \pi 0 / 5}+e^{i 2 \pi 1 / 5}+e^{-i 2 \pi 1 / 5}=r$, а для представления $T_{2 u}$ равна $1-r$. В классе поворотов $20 C_{3}$ в ноль будет обращаться шпур для трехмерных представлений $T_{1 u}$ и $T_{2 u}$. Одномерное представление $A_{g}$ и в этом случае сохраняет шпур равным 1. Для пятимерного представления $H_{g}$ вычисление суммы диагональных элементов приводит к выражению $\sum_{k=1}^{5} e^{i 2 \pi k / 3}=-1$. Повороты на угол $\pi$ для класса сопряженных элементов $15 C_{2}$ приведены в следующей колонке. Они состоят из чередующихся +1 и -1 , либо нулей в случае с $G$. Во второй части таблицы характеры для антисимметричных представлений с индексом $u$ меняют знак. Ортогональность представлений задается через формулу скалярного произведения характеров [5] (здесь размер класса представлен в виде множителя $\left.\alpha_{p}: \alpha_{E}=1, \alpha_{C_{5}}=12, \alpha_{C_{3}}=20 \ldots\right)$ :

$$
\left(\chi_{a}, \chi_{b}\right)=\sum_{p} \alpha_{p} \chi_{a}(p) \chi_{b}(p) .
$$

Так как таблица имеет размер 10 на 10, то она полна. По горизонтали расположены все десять классов преобразований. С учетом размерности классов самосопряженных элементов, полное число элементов $2(1+12+12+20+15)=120$ как и число элементов группы. Число неприводимых представлений тоже 10 (с учетом четности). Причем квадраты размерностей
Таблица 1. Таблица характеров для неприводимых представлений группы $I_{h}$ в случае усеченного икосаэдра, $r=(1+\sqrt{5}) / 2$ и является корнем уравнения $r^{2}=r+1$. Индекс $g$ обозначает четное представление, $u-$ нечетное относительно центральной инверсии $P$

\begin{tabular}{l|l|l|l|l|l|l|l|l|l|l}
\hline$I_{h}$ & $E$ & $12 C_{5}$ & $12 C_{5}^{2}$ & $20 C_{3}$ & $15 C_{2}$ & $P$ & $12 S_{10}^{3}$ & $12 S_{10}$ & $20 S_{3}$ & $15 \sigma_{v}$ \\
\hline$A_{g}$ & +1 & +1 & +1 & +1 & +1 & +1 & +1 & +1 & +1 & +1 \\
\hline$T_{1 g}$ & +3 & $+r$ & $1-r$ & 0 & -1 & +3 & $r$ & $1-r$ & 0 & -1 \\
\hline$T_{2 g}$ & +3 & $1-r$ & $r$ & 0 & -1 & +3 & $1-r$ & $r$ & 0 & -1 \\
\hline$G_{g}$ & +4 & -1 & -1 & +1 & 0 & +4 & -1 & -1 & +1 & 0 \\
\hline$H_{g}$ & +5 & 0 & 0 & -1 & +1 & +5 & 0 & 0 & -1 & +1 \\
\hline$A_{u}$ & +1 & +1 & +1 & +1 & +1 & -1 & -1 & -1 & -1 & -1 \\
\hline$T_{1 u}$ & +3 & $+r$ & $1-r$ & 0 & -1 & -3 & $-r$ & $r-1$ & 0 & +1 \\
\hline$T_{2 u}$ & +3 & $1-r$ & $r$ & 0 & -1 & -3 & $r-1$ & $-r$ & 0 & +1 \\
\hline$G_{u}$ & +4 & -1 & -1 & +1 & 0 & -4 & +1 & +1 & -1 & 0 \\
\hline$H_{u}$ & +5 & 0 & 0 & -1 & +1 & -5 & 0 & 0 & +1 & -1
\end{tabular}

неприводимых представлений $A, T_{1}, T_{2}, G, H$ дают размерность группы вращений: $1^{2}+3^{2}+3^{2}+4^{2}+5^{2}=60$. С учетом четности сумма квадратов вновь становится 120 . В колонке единичного преобразования $E$ прослеживается размерность пространства каждого из неприводимых представлений. Размещая в каждой вершине усеченного икосаэдра атом углерода, получаем модель фуллерена $C_{60}$. Считая, что в каждом таком узле сидит по одному $\pi$-электрону, получим 60 состояний электронов в молекуле. Если перед представлением ставить число кратности состояния, то в фуллерене реализуется следующая композиция неприводимых представлений: $1 A_{g}, 2 T_{1 u}, 2 T_{2 u}, 1 T_{1 g}, 1 T_{2 g}, 2 G_{u}, 2 G_{g}, 2 H_{u}$, $3 H_{g}$. С учетом кратности и размерности представлений получим в точности $1+2 \times 3+2 \times 3+3+3+$ $2 \times 4+2 \times 4+2 \times 5+3 \times 5=60$ состояний.

Направим ось $z$ через центр усеченного икосаэдра и центр грани пятиугольника, как представлено на рис. 2. Определим декартову тройку осей так, чтобы ось $x$ лежала в плоскости симметрии фигуры-шаблона из 6 точек, выделенных цветом и двойной линией. Данная фигура при повороте вокруг оси $z$ на угол, кратный $2 \pi / 5$, покрывает все точки тела в верхней части полупространства. Задав только 6 вершин на указанном фрагменте усеченного икосаэдра, с помощью двух квантовых чисел, задающих свойства симметрии относительно оси пятого порядка и инверсии относительна центра икосаэдра, можно описать все состояния системы. Первое квантовое число обозначим буквой $m$, второе буквой $p$. Представления $A_{g}, T_{1 g}, T_{2 g} \ldots$ симметричны относительно инверсии, и квантовое число равно $p=1$. Для представлений $T_{1 u}, T_{2 u}, G_{u}, H_{u}$ реализуется антисимметричная комбинация значений волновых функций отно- 
сительно операции инверсии, и квантовое число равно $p=-1$. Квантовое число $m$ задает множитель перед волновой функцией $\eta^{m}=\exp \left(i \frac{2 \pi}{5} m\right)$, описывающий осцилляции волновой функции при каждом повороте шаблона. Всего существует пять нетривиальных вариантов или состояний (с $m=0, \pm 1, \pm 2$ ). Неприводимые представления отличаются набором комбинаций состояния числа $m$. Представление $A_{g}$ содержит только состояние с $m=0$. Представление $H$ включает все возможные состояния. Представление $G$ задается четырьмя проекциями с $m= \pm 1, \pm 2$, представление $T_{1}$ - тремя проекциями с $m=0, \pm 1$. Самое необычное представление $T_{2}$, в нем нет проекций \pm 1 (т. е. $m=0, \pm 2$ ). Таким образом, число $m$ соответствует проекции орбитального момента $L=2$ на ось $z$ для неприводимого представления группы вращений $\mathrm{SO}(3)$ [4].

Использование симметрии относительно оси пятого порядка открывает возможности аналитического исследования спектра собственных значений системы фуллерена. Гамильтониан для системы из $60 \pi$-электронов в модели молекулярных орбиталей Хюккеля приводит к поиску спектра квадратной матрицы ранга 60. Как известно, уравнения выше пятой степени неразрешимы. Подход Денга и Янга [4] позволяет понизить ранг исследуемых матриц до шести. Рассмотрим некоторое представление, например, $H_{u}$. Это пять разных волновых функций для пятикратно вырожденного состояния системы. Это означает, что в исходной матрице есть 5 равных собственных значений. Они соответствуют различным проекциям момента на ось $z$, задаваемым квантовым числом $m$. Тогда, разделяя состояния по числам $m, p$, фактически снимаем кратные корни для оставшейся в проекции системе (без учета случайного вырождения). Так, случай $p=1, m=0$ дает 6 корней характеристического уравнения: один $A_{g}$ как корень линейного уравнения, два корня $T_{1 g}, T_{2 g}$ как смешанные в квадратном уравнении, и три корня, сцепленные между собой в рамках кубического уравнения для трех различных неприводимых представлений $H_{g}$ (для представления $G_{g}$ проекции с нулевым $m$ нет). Аналогично анализ случая другой симметрии $(p=-1, m=0)$ дает другие 6 корней: два корня для представления $H_{u}$, два корня для представления $T_{1 u}$ и два корня для $T_{2 u}$. Все корни в каждой паре сцеплены между собой и могут быть приведены к квадратным алгебраическим уравнениям. Таким образом, система разрешима в радикалах (приводима в рамках идеологии Галуа). Недостающие элементы спектра могут быть вычислены (в случае представления $G)$ при ненулевых значениях квантового числа $m$. Понижение размерности системы за счет устранения кратных корней при вычислении спектра собственных значений гамильтониана - сильный бонус теории представлений. Поскольку $m$ принимает пять значений, а $p$ два, то ранг итоговой матрицы гамильтониана понижается до шести. Как оказалось, максимальная сложность, возникающая при разложении на множители характеристического уравнения, это уравнения четвертого порядка
$2 T_{1 u} \oplus 2 T_{2 u}$. Данная четверка понижается в конечном итоге до двух квадратных уравнений с трансцендентными коэффициентами. Приведенные свойства симметрии относительно оси пятого порядка расшифровывают возникающий список неприводимых представлений с учетом кратности: $1 A_{g}, 2 T_{1 u}, 2 T_{2 u}, 1 T_{1 g}, 1 T_{2 g}, 2 G_{u}, 2 G_{g}$, $2 H_{u}, 3 H_{g}$.

При численном и аналитическом подходах роль диагональных членов гамильтониана различна. При численном анализе для систем большой размерности работают в пространствах Крылова, поэтому спектр матрицы необходимо сдвинуть, чтобы он был положительно определен. Достигается это с помощью диагональной матрицы заданного значения, которое будет в дальнейшем учтено. Диагональная матрица сдвигает спектр, но не трогает собственные векторы. В противоположность этому в аналитическом подходе устраняются диагональные члены гамильтониана с помощью дополнительной диагональной матрицы. Исследуемый спектр содержит только эффекты от коллективных свойств молекулы, возникающих при обобществлении $\pi$-электронов. Сдвиг энергии спектра может быть устранен непосредственно из экспериментальных данных.

Так как число вершин равно 60, а число ребер равно 90, то шаблон состоит из 6 вершин и 9 ребер (рис. 2). Ребра, входящие в шаблон, на рисунке помечены звездочками. Если бы все ребра в молекуле имели равную длину, то гамильтониан в модели молекулярных орбиталей имел бы следующий вид:

$$
\begin{aligned}
& \langle\psi \mid H \psi\rangle=10\left\{\left|\psi_{1}-\psi_{1} \eta^{m}\right|^{2}+\left|\psi_{1}-\psi_{2}\right|^{2}+\left|\psi_{2}-\psi_{3}\right|^{2}\right. \\
& +\left|\psi_{2}-\psi_{4}\right|^{2}+\left|\psi_{3}-\psi_{5}\right|^{2}+\left|\psi_{4}-\psi_{6}\right|^{2}+\left|\psi_{5}-\psi_{6}\right|^{2} \\
& \left.+\left|\psi_{4}-\psi_{3} \eta^{m}\right|^{2}+\left|\psi_{6}-\psi_{5} p \eta^{3 m}\right|^{2}-3 \sum_{6}\left|\psi_{i}\right|^{2}\right\}
\end{aligned}
$$

Последнее слагаемое устраняет диагональные члены, шаблон применяется 10 раз с учетом симметрии относительно оси пятого порядка и инверсии. Там, где ребро соединяет вершину шаблона с копией, полученной поворотом, возникает множитель $\eta^{m}$. В случае, когда ребро уходит в нижнюю полусферу, то соответствующий коэффициент отвечает повороту на тройной угол и инверсии шаблона.

Как известно, в молекуле фуллерена длина ребер внутри пятиугольника и ребер, соединяющих смежные пятиугольники, различна. В гамильтониане эту разницу будем учитывать с помощью параметра $\alpha$. Когда он равен единице, то получим гамильтониан (2). В противном случае поправочный множитель для силы связи зависит от длины ребра и равен $\alpha$ либо $\beta=(2-\alpha)$ соответственно в зависимости от типа связи (на рисунке звездочки разных типов связи помечены цветом). Для задачи на собственное значение будем использовать матричный вид гамильтониана, применив 
к нему унитарное преобразование $U$. Искомый детерминант матрицы после преобразования имеет вид $P=\operatorname{det}(\hat{H}-\lambda E)=\left|U^{+} H U-\lambda E\right|=0$ :

$$
\begin{array}{cccccc}
-\alpha 2 c_{m}-\lambda & -\beta & 0 & 0 & 0 & 0 \\
-\beta & -\lambda & -\alpha \sqrt{2} & 0 & 0 & 0 \\
0 & -\alpha \sqrt{2} & -\beta c_{m}-\lambda & \beta s_{m} & -\alpha & 0 \\
0 & 0 & \beta s_{m} & \beta c_{m}-\lambda & 0 & -\alpha \\
0 & 0 & -\alpha & 0 & -\alpha-\beta p c_{3 m}-\lambda & \beta p s_{3 m} \\
0 & 0 & 0 & -\alpha & \beta p s_{3 m} & \alpha+\beta p c_{3 m}-\lambda
\end{array} \mid
$$

Здесь введены обозначения $c_{m}=\cos \left(\frac{2 \pi m}{5}\right)$, $s_{m}=\sin \left(\frac{2 \pi m}{5}\right)$, а $\eta^{m}+\eta^{-m}=2 c_{m}$. Матрица унитарного преобразования состоит из квадратных матриц по диагонали:

$$
\begin{gathered}
U=\left(\begin{array}{ccc}
E & 0 & 0 \\
0 & T & 0 \\
0 & 0 & T
\end{array}\right), \quad E=\left(\begin{array}{cc}
1 & 0 \\
0 & 1
\end{array}\right), \\
T=\left(\begin{array}{ll}
1 / \sqrt{2} & +i / \sqrt{2} \\
1 / \sqrt{2} & -i / \sqrt{2}
\end{array}\right) .
\end{gathered}
$$

Для четных состояний волновой коллективной функции получим следующие корни характеристического уравнения в зависимости от типа неприводимого представления:

$$
\begin{gathered}
1 A_{g}: \lambda+2+\alpha=0, \\
1 T_{1 g}: \lambda-2=-\frac{\alpha}{2}(\sqrt{5}+1)=2 \alpha c_{3}, \\
1 T_{2 g}: \lambda-2=\frac{\alpha}{2}(\sqrt{5}-1)=2 \alpha c_{1}, \\
2 G_{g}: \lambda^{2}-\alpha \lambda+\left(-4+4 \alpha-2 \alpha^{2}\right)=0, \\
3 H_{g}: \lambda^{3}+2 \lambda^{2}+\left(-4+8 \alpha-6 \alpha^{2}\right) \lambda \\
+\left(-8+16 \alpha-12 \alpha^{2}+\alpha^{3}\right)=0 .
\end{gathered}
$$

Здесь

$$
c_{1}=\frac{\sqrt{5}-1}{4}, \quad c_{3}=-\frac{\sqrt{5}+1}{4} .
$$

Для нечетных мод набор корней в зависимости от типа неприводимых представлений выглядит следующим образом:

$$
\begin{aligned}
2 T_{1 u}: \lambda^{2} & +\frac{\lambda \alpha}{2}(3+\sqrt{5})+\frac{\alpha^{2}(-5+3 \sqrt{5})}{2} \\
& +\alpha(5-\sqrt{5})-4=0, \\
2 T_{2 u}: \lambda^{2}+ & \frac{\lambda \alpha}{2}(3-\sqrt{5})+\frac{\alpha^{2}(-5-3 \sqrt{5})}{2} \\
& +\alpha(5+\sqrt{5})-4=0, \\
2 G_{u}: \lambda^{2}-\alpha \lambda-4=0, & \\
2 H_{u}: & \lambda^{2}-\alpha \lambda-4+6 \alpha-3 \alpha^{2}=0 .
\end{aligned}
$$

Результаты коррелируют с полученными в работе [6]. Собственные векторы $\hat{\psi}$ матрицы (3) находятся аналитически, однако из-за сложности формул выражения для них опускаем. Искомые волновые функции есть $\psi=U \hat{\psi}$.

Расчет спектра энергий (табл. 2) был выполнен на основе аналитических формул, в которых был устранен произвол по сдвигу спектра и параметру $\alpha$ (об этом ниже). Поскольку некоторые представления возникают несколько раз, то в скобках указан текущий номер повтора представления. При каждом появлении коллективная мода, к которой принадлежит мультиплет уровней, соответствующий данному представлению, имеет некоторый уникальный орбитальный момент моды. С ростом номера мультиплета за небольшими исключениями растет орбитальный момент. С изменением орбитального момента естественно изменяется и энергия уровней за счет изменения вращательной компоненты кинетической энергии (в этой системе вырождения энергии по орбитальному моменту, как у атома водорода, нет).

Критическим отличием от неприводимых представлений группы вращений $\mathrm{SO}(3)$ является то, что число проекций на ось $z$ ограничено числом 5, в то время как для классического орбитального момента $L$ их число равно $2 L+1$. Поэтому интересно провести сравнение с группой вращения в непрерывном пространстве. В квантовой механике учет симметрии, связанной с группой вращения, приводит к факторизации волновой функции на радиальную и угловую части. Угловая волновая функция выражается посредством сферических функций вида $Y_{l m}(\vartheta, \varphi) \propto P_{l}^{|m|}(\cos \vartheta) e^{i m \varphi}$. Угловая часть волновой функции определяется набором двух квантовых чисел - орбитальным моментом $l$ и проекцией на ось $m$. Рассматривая данные функции на дискретном наборе преобразований - наборе поворотов для усеченного икосаэдра, получим таблицу характеров, которая обладает уникальным узором. Для каждого элемента группы $C_{\alpha}(\alpha-$ угол поворота) в таблице характеров значение в строке с заданным орбитальным моментом $l$ дается простой формулой [7]:

$$
\chi_{l}\left(C_{\alpha}\right)=\sin \left((2 l+1) \frac{\alpha}{2}\right)\left(\sin \left(\frac{\alpha}{2}\right)\right)^{-1} .
$$

В случае группы поворотов усеченного икосаэдра система характеров ортогональна и полна, и по ней можно раскладывать любой новый характер. То есть можно получить правила разложения орбитального момента в прямую сумму неприводимых представлений икосаэдра. Результаты вычислений сведены в табл. 3 (для $L \leq 2$ возникает однозначное соответствие.

Обращаясь к табл. 3, можно заметить, что, начиная с орбитального момента, равного трем, состояние квантовой системы задается прямой суммой нескольких неприводимых представлений группы икосаэдра. Каждому неприводимому представлению отвечает свое значение уровня энергии для всей подсистемы подуровней. Так, орбитальный момент, равный 5, отвечает трем 
Таблица 2. Заполнение уровней энергии для коллективных мод фуллерена $\pi$-электронами

\begin{tabular}{|c|c|c|c|c|c|}
\hline $\begin{array}{c}\text { Номер } \\
\text { мультиплета }\end{array}$ & Представление & $\begin{array}{c}\text { Размерность } \\
\text { представления }\end{array}$ & $\begin{array}{c}\text { Орбитальный } \\
\text { момент } L\end{array}$ & $\begin{array}{c}\text { Энергия } \\
\text { уровней, eV }\end{array}$ & $\begin{array}{c}E \text { в потенциале } \\
U, \mathrm{eV}\end{array}$ \\
\hline 1 & $A_{g}(1)$ & 1 & 0 & -12.7 & -12.05 \\
\hline 2 & $T_{1 u}(1)$ & 3 & 1 & -11.60 & -11.73 \\
\hline 3 & $H_{g}(1)$ & 5 & 2 & -10.73 & -11.08 \\
\hline 4 & $T_{2 u}(1)$ & 3 & 3 & -9.69 & -10.13 \\
\hline 5 & $G_{u}(1)$ & 4 & 3 & -9.45 & \\
\hline 6 & $G_{g}(1)$ & 4 & 4 & -8.36 & -8.87 \\
\hline 7 & $H_{g}(2)$ & 5 & 4 & -8.26 & \\
\hline 8 & $H_{u}(1)$ & 5 & 5 & -7.61 & -7.31 \\
\hline 9 & $T_{1 u}(2)$ & 3 & 5 & -5.84 & \\
\hline 10 & $T_{1 g}(1)$ & 3 & 6 & -5.37 & -5.44 \\
\hline 11 & $H_{g}(3)$ & 5 & 6 & -3.96 & \\
\hline 12 & $T_{2 u}(2)$ & 3 & 5 & -3.69 & \\
\hline 13 & $H_{u}(2)$ & 5 & 7 & -3.27 & -3.30 \\
\hline 14 & $G_{g}(2)$ & 4 & 6 & -2.52 & \\
\hline 15 & $G_{u}(2)$ & 4 & 7 & -1.43 & \\
\hline 16 & $T_{2 g}(1)$ & 3 & 8 & -1.32 & \\
\hline
\end{tabular}

Примечание. Мультиплеты разделены по неприводимым представлениям. В скобках указана кратность повтора представления в системе по мере заполнения. Также приведены кратность вырождения уровня (размерность представления), орбитальный момент коллективной моды и энергия в электрон-вольтах. Правая колонка дает уровни энергии потенциала (13) для основного состояния при изменении орбитального квантового числа.

Таблица 3. Разложение орбитального момента в прямую сумму неприводимых представлений икосаэдра. Более высокие моменты можно найти в [8]

\begin{tabular}{c|c|c}
\hline $\begin{array}{c}\text { Орбитальный } \\
\text { момент }\end{array}$ & Сумма представлений & $\begin{array}{c}\text { Сумма } \\
\text { размерностей }\end{array}$ \\
\hline$L=0$ & $A_{g}$ & 1 \\
\hline$L=1$ & $T_{1 u}$ & 3 \\
\hline$L=2$ & $H_{g}$ & 5 \\
\hline$L=3$ & $G_{u} \oplus T_{2 u}$ & $4+3$ \\
\hline$L=4$ & $G_{g} \oplus H_{g}$ & $4+5$ \\
\hline$L=5$ & $H_{u} \oplus T_{1 u} \oplus T_{2 u}$ & $5+3+3$ \\
\hline$L=6$ & $G_{g} \oplus H_{g} \oplus A_{g} \oplus T_{1 g}$ & $4+5+1+3$ \\
\hline$L=7$ & $G_{u} \oplus\left(H_{u} \oplus T_{1 u} \oplus T_{2 u}\right)$ & $4+11$ \\
\hline$L=8$ & $\left(H_{g} \oplus G_{g}\right) \oplus H_{g} \oplus T_{2 g}$ & $9+5+3$
\end{tabular}

подсистемам из пяти линий $\left(H_{u}\right)$ и двух триплетов $\left(T_{1 u}\right.$, $T_{2 u}$ ) - всего 11 уровней. Однако подсистема электронов с квантовым числом 6 для орбитального момента имеет не 13 подуровней, а только 12: $\left(T_{1 g}, H_{g}, G_{g}\right.$ т.е. $=3+5+4)$. Подсистема $A_{g}$ не получила реализацию. Также для квантового числа $L=7$ реализуются две подсистемы с общей размерностью пространства в 9 уровней $\left(H_{u} \oplus G_{u}\right.$, вместо пятнадцати). То есть система с точечной симметрией икосаэдра допускает нарушение правила - число проекций момента $L$ равно $2 L+1$, по крайней мере для некоторых высоких моментов. Так, для момента $L=8$ вообще реализуется только одно неприводимое представление $T_{2 g}$ из четырех возможных. Даже там, где число подуровней равно $2 L+1$, угловая часть волновой функциии имеет вид, отличный от сферических функций. Например, для представления $T_{2 u}$ волновые функции были получены в виде $Y_{30}$ и $\pm \sqrt{2 / 5} Y_{3, \mp 3}+\sqrt{3 / 5} Y_{3, \pm 2}$. Так как ось пятого порядка вводит алгебру целых в кольцах по модулю 5, то в формулах присутствует одновременно зависимость от азимутальных углов $2 \varphi$ и $3 \varphi$ (ввиду $3=2 \bmod (5))$. Однако коэффициенты разложения неприводимого представления в симметрии усеченного икосаэдра по представлениям сферических функций требуют дополнительного внимания (часть списка функций можно найти в работах [2] (на с. 83) или [9]). 


\section{3. Особенности симметрии усеченного икосаэдра}

В качестве примера модели усеченного икосаэдра мы используем молекулу фуллерена $\mathrm{C}_{60}$. Обычно $[4,10]$, получив зависимости от параметра $\alpha$ для энергии, следующие из выражений (4),(5), исследуют графически полученные зависимости, оставив его свободным. Однако данные о молекуле фуллерена, приведенные в таблице физических констант в [2], позволяют изменить стратегию исследования. Зафиксировав трехмерную геометрию молекулы, будем варьировать параметр $\alpha$, чтобы найти минимальную погрешность в аппроксимации волновой функции для реализации представления $T_{1 u}$ в виде $\psi=z=\cos \theta$ (табл. 4). Расположение вершин усеченного икосаэдра таково, что рисунок заполнения точек на поверхности сферы, описанной вокруг усеченного икосаэдра, неравномерный по азимутальному и полярному углам. Это снимает вырождение положений вершин, присущее икосаэдру, и позволяет найти различие волновых функций, возникающее из-за вариации силы связей, соответствующих связям пентагона и гексагона. Наилучшее приближение в смысле наименьшего среднеквадратичного отклонения позволяет исключить параметр $\alpha$ даже в рамках метода молекулярных орбиталей Хюккеля без решения точной задачи квантовой химии.

Для реализации данной программы были использованы два подхода. В первом из них были взяты данные о геометрии молекулы $\mathrm{C}_{60}$ из отчета об исследовании этой молекулы на основе программ по квантовой химии [11]. Длина ребра пентагона $R_{60}=1.43737 \AA$, а длина ребра смежных гексагонов $R_{30}=1.38195 \AA$. Данные получены в результате усреднения по всему набору из 60 и 30 ребер соответственно. Это результат соответствует результатам по рентгеновской дифракции $\left(R_{30}=1.388(9) \AA, R_{60}=1.432(5) \AA\right)$ [12]. Сами данные флуктуируют из-за машинного представления координат с минимальной машинной точностью. Гамильтониан задаем в виде матрицы $60 \times 60$ элементов, моделируя силу связи между ближайшими соседями двумя параметрами $c_{60}, c_{30}$, а диагональные элементы положим равными $c_{0}=1$. Схема ближайших соседей соответствует диаграмме Шлегеля [13] для фуллерена $\mathrm{C}_{60}$.

Собственные векторы и числа для данной матрицы анализировались в пространствах Крылова, выбирая старшие 30 или младшие 30 (для обратной матрицы) по величине собственного числа. Отобрав нужное неприводимое представление $T_{1 u}$ по значению энергии из табл. 4, с помощью собственного вектора задаем волновую функцию в каждой вершине молекулы. Посредством специальной процедуры фиксируем разворот молекулы в трехмерном пространстве и сравниваем с тремя возможными вариантами реализации представления, полученными на основе теории представлений. Метод псевдообратной матрицы [14] (в простейшем случае - линейная регрессия) позволяет идентифицировать представление и найти ошибку - невязку полученного решения, по методу наименьших квадратов. При фиксированной величине $c_{60}=c_{0} / 10$ минимальная невязка обнаружилась для значения связи в шестиугольнике $c_{30}=c_{0} / 8.5512$. Из системы уравнений (где $\eta-$ фактор шкалы энергии, на невязку не влияет)

$$
\begin{aligned}
& c_{60}=\eta \alpha, \\
& c_{30}=\eta \beta
\end{aligned}
$$

следует, что $\alpha=\frac{2 c_{60} / c_{30}}{c_{60} / c_{30}+1}=0.9219$ и $\beta=(2-\alpha)>1$.

Во втором подходе вместо численного решения матрицы в $60 \times 60$ элементов мы используем аналитику, развитую в предыдущем разделе. Данный подход понижает ранг матрицы в задаче на собственные значения до 6. Также аналитически изучали геометрию задачи. Отметим, что в усеченном икосаэдре минимальный угол поворота $\vartheta$, который приводит к отображению осей, проведенных из центра тела к центрам правильных пятиугольников, на себя, находится из равенства $\cos \vartheta=1 / \sqrt{5}$. Введем вспомогательный угол $\vartheta_{1}$, который вычисляется по формуле

$$
\tan \left(\vartheta_{1}\right)=\frac{\sin (\vartheta / 2)}{\left(R_{30} / R_{60}\right) \sin (\pi / 5)+\cos (\vartheta / 2)}
$$

Тогда можно найти азимутальный $\phi_{k}$ и полярный углы $\theta_{k}$ для вершины с номером $k$. Отсчитываем азимутальный угол от нулевого меридиана, заданного точками 1 и 2. Для них полярные углы равны $\theta_{1}=\vartheta_{1}$ и $\theta_{2}=\vartheta-\vartheta_{1}$ соответственно. Точки 4 и 6 отличаются от точек 3 и 5 знаком угла $\phi$. Поэтому достаточно найти углы точек 3 и 5. Для этих точек азимутальные углы и полярные углы удовлетворяют системе четырех уравнений:

$$
\begin{gathered}
\sin \theta_{3} \sin \left|\phi_{3}\right|=\sin \vartheta_{1} \sin (2 \pi / 5) \\
\sin \theta_{5} \sin \left|\phi_{5}\right|=\sin \vartheta_{1} \sin (\pi / 5) . \\
\cos \theta_{2} \cos \theta_{3}+\sin \theta_{2} \sin \theta_{3} \cos \phi_{3}=1-2 \sin ^{2} \vartheta_{1} \sin ^{2} \frac{\pi}{5} \\
\cos \theta_{2} \cos \theta_{5}+\sin \theta_{2} \sin \theta_{5} \cos \phi_{5}=1-2 \sin ^{2} \vartheta_{1} \sin ^{2} \frac{2 \pi}{5}
\end{gathered}
$$

Остальные вершины фуллерена также могут быть описаны в терминах полярных и азимутальных углов с помощью операций поворота шаблона вокруг оси ZO пятого порядка и операции инверсии вокруг центра $O$. Большая точность нахождения полярных и азимутальных углов вершин позволяет достичь на 2 порядка меньшей невязки для $T_{1 u}$, чем при численном решении матрицы гамильтониана в $60 \times 60$ элементов. Так, при $\alpha=0.92205$ удалось достичь невязки $10^{-7}$ для волновых функций неприводимого представления $T_{1 u}$ по сравнению со сферическими функциями с орбитальным моментом $L=1$. Выше была получена константа $\alpha=0.92190$ (когда данные о положении вершин содержали численный шум). Как видим, различие 
всего на единицу в четвертом знаке после запятой ведет к росту точности аппроксимации. Таким образом, из геометрии пятиугольников и шестиугольников следует единственное значение параметра $\alpha$, обеспечивающее теоретически обоснованный вид волновой функции представления $T_{1 u}$ по поверхности молекулы фуллерена. Остаются в модели только две неопределенных константы - диагональ гамильтониана и шкала энергии для недиагональных элементов, которые легко определить из известных экспериментальных данных. Диагональные элементы определяют сдвиг спектра системы, который может быть найден из данных по ионизации, данные по спектру поглощения позволяют исключить константу $\eta$. В результате можно найти уровни энергии, их значения в электрон-вольтах приведены в табл. 2. Табличные данные получены в предположении, что $R_{30}=1.39 \AA$ и $R_{60}=1.43 \AA$, заданных в рамках указанной точности. Для этих расстояний между атомами $\alpha=0.927086$ (невязка $=10^{-9}$ ). Последний заполненный уровень принадлежит неприводимому представлению $H_{u}$ с орбитальным моментом $L=5$. Все уровни этого представления заполнены 10 электронами, энергия ионизации с этого уровня равна $7.61 \mathrm{eV}$. Экспериментальные данные об уровнях энергии нейтрального фуллерена извлечены из работ $[15,16]$. Используя одну из линий поглощения, равную $2.24 \mathrm{eV}$, можно воспроизвести всю структуру уровней. Разброс в положении линий в спектре поглощения, описывающих переходы между уровнями энергии, не превышают $0.1 \mathrm{eV}$.

Для описания волновых функций неприводимых представлений установим соответствие последних со сферическими волновыми функциями [17]. По определению

$$
\begin{gathered}
Y_{l m}=(-1)^{(m+|m|) / 2} i^{l} c_{l,|m|} P_{l}^{|m|}(\cos \theta) e^{i m \varphi}, \\
c_{l,|m|}=\sqrt{\frac{2 l+1}{4 \pi} \frac{(l-|m|) !}{(l+|m|) !}} .
\end{gathered}
$$

Здесь $P_{l}^{|m|}$ - присоединенные полиномы Лежандра (при $m=0$ - полиномы Лежандра). В табл. 4 мы использовали наилучшее приближение волновых функций, полученных аналитически, с помощью комбинаций сферических функций нужной симметрии. В последней колонке приведены среднеквадратичная ошибка и нормировочная константа для вещественных решений. Отметим, что ошибка аппроксимации не зависит от выбора компоненты (подуровня) рассматриваемого представления. Если ввести обозначение для неприводимого представления в виде $N X_{y}(L)(N-$ номер, $L-$ орбитальный момент представления), то наилучшее приближение у двух представлений $3 T_{1 u}(2)$ и $10 T_{1 g}(6)$. Наихудшая аппроксимация у представлений $6 G_{g}(4), 7 H_{g}(4)$ и $9 T_{1 u}(5)$. Многие волновые функции описываются посредством двух и даже трех сферических функций, множители выражаются с машинной точностью через радикалы. Данные наборы функций обеспечивают ортогональность пространства каждого из представлений. Как и в случае симметрии икосаэдра, у усеченного икосаэдра представление волновых функций через сферические функции - удобный инструмент, дающий весьма достоверные результаты. Аналитический вид гамильтониана вообще не содержит информации о координатах вершин, сохраняя только свойства точечной симметрии и список ближайших соседей.

Ортогональность собственных векторов с разными собственными значениями возникает вследствие симметричности вещественных матриц гамильтониана. После привязки вершин к координатной сетке с учетом симметрии икосаэдра вычисляется волновая функция от координат вершин. И теперь вместо точечной симметрии на конечном множестве вершин речь идет о геометрии в непрерывном трехмерном пространстве и группах вращений для сферы, описанной вокруг вершин усеченного икосаэдра. Ясно, что результаты, полученные по точечной модели, могут быть отображены в пространство с непрерывной метрикой и аналитически продолжены на окрестности заданных вершин усеченного икосаэдра. Однако ничто не гарантирует, что образ в пространстве сферических функций и оригинал - это две идентичные формы на множестве вершин. Как ни удивительно, но существует полное совпадение форм для некоторых неприводимых представлений, которое достигается подбором параметра $\alpha$, управляющего изменением силы связи с изменением дистанции между атомами. Для остальных отображение - это наилучшее приближение данным набором функций. Учитывая небольшую погрешность приближения и то обстоятельство, что физические свойства оптических переходов связаны именно с разложением по сферическим гармоникам, данная таблица дает хороший инструментарий для анализа оптических свойств дипольных (квадрупольных) переходов, а также может использоваться для вывода сечений возбуждения электронным ударом.

\section{4. Физические свойства электронных переходов}

В данном разделе рассмотрим переход между уровнями неприводимых представлений $8 H_{u}(5)$ и $10 T_{1 g}(6)$. Поскольку уровень $\left(H_{u}\right)^{10}$ заполнен полностью, то считается, что его симметрия $A_{g}$. Когда один электрон покидает этот уровень, то симметрия принимает значение $H_{u}$. Поэтому с учетом заселения состояния $T_{1 g}$ общая симметрия системы в конфигурации $\left(H_{u}\right)^{9}\left(T_{1 g}\right)^{1}$ описывается прямым произведением неприводимых представлений: $H_{u} \otimes T_{1 g}$. Поскольку характер прямого произведения представлений равен произведению характеров представлений, входящих в произведение поэлементно $\chi_{H_{u} \otimes T_{1 g}}(g)=\chi_{H_{u}}(g) \chi_{T_{1 g}}(g)$, то из их таблицы характеров следует, что это прямое 
Таблица 4. Наилучшее приближение нормированных волновых функций 10 первых неприводимых представлений сферическими функциями. Для удобства приведены также реальные и мнимые части волновых функций с нормировочным множителем; здесь $x^{2}+y^{2}+z^{2}=1, z=\cos \vartheta$

\begin{tabular}{|c|c|c|c|}
\hline Представление & Функция $\Psi$ & $\operatorname{Re} \Psi / \beta, \operatorname{Im} \Psi / \beta$ & Примечание \\
\hline $1 A_{g}(0)$ & $Y_{00}$ & 1 & $\beta=\sqrt{1 / 4 \pi}$ \\
\hline $2 T_{1 u}(1)$ & $Y_{10}$ и $Y_{1,1}, Y_{1,-1}$ & $z$ и $(x, y)$ & $\begin{array}{l}\beta=\sqrt{3 / 4 \pi} \\
\text { err }=2 \times 10^{-9}\end{array}$ \\
\hline $3 H_{g}(2)$ & $Y_{20} ; Y_{2,1}, Y_{2,-1} ; Y_{2,2}, Y_{2,-2}$ & $\left(3 z^{2}-1\right) / \sqrt{3} ;(2 z x, 2 z y) ;\left(x^{2}-y^{2}, 2 x y\right)$ & $\begin{array}{l}\beta=\sqrt{15 / 16 \pi} \\
\text { err }=3 \times 10^{-3}\end{array}$ \\
\hline $4 T_{2 u}(3)$ & $Y_{30} ; \sqrt{2 / 5}\left(\mp Y_{3, \pm 3}+\sqrt{3 / 2} Y_{3, \mp 2}\right)$ & $\begin{array}{l}\left(5 z^{3}-3 z\right) ; x\left(x^{2}-3 y^{2}\right)+3 z\left(x^{2}-y^{2}\right) \\
y\left(3 x^{2}-y^{2}\right)-6 x y z\end{array}$ & $\begin{array}{l}\beta=\sqrt{7 / 16 \pi} \\
\text { err }=7 \times 10^{-3}\end{array}$ \\
\hline $5 G_{u}(3)$ & $\sqrt{3 / 5}\left(\mp Y_{3, \pm 3}-\sqrt{2 / 3} Y_{3, \mp 2}\right) ; Y_{3,1}, Y_{3,-1}$ & $\begin{array}{l}x\left(x^{2}-3 y^{2}\right)-2 z\left(x^{2}-y^{2}\right) \\
y\left(3 x^{2}-y^{2}\right)+4 x y z \\
\left(x\left(5 z^{2}-1\right), y\left(5 z^{2}-1\right)\right)\end{array}$ & $\begin{array}{l}\beta=\sqrt{21 / 32 \pi} \\
\text { err }=5 \times 10^{-3}\end{array}$ \\
\hline $6 G_{g}(4)$ & $\begin{array}{l}\sqrt{8 / 15}\left(\sqrt{7 / 8} Y_{4, \pm 1} \mp Y_{4, \mp 4}\right) \\
\sqrt{1 / 15}\left(\sqrt{14} Y_{4, \pm 2} \mp Y_{4, \mp 3}\right)\end{array}$ & $\begin{array}{l}x z\left(7 z^{2}-3\right)+\left(x^{4}+y^{4}-6 x^{2} y^{2}\right) \\
y z\left(7 z^{2}-3\right)-4 x y\left(x^{2}-y^{2}\right) \\
\left(7 z^{2}-1\right)\left(x^{2}-y^{2}\right)-x z\left(x^{2}-3 y^{2}\right) \\
\left(7 z^{2}-1\right)(2 x y)+y z\left(3 x^{2}-y^{2}\right)\end{array}$ & $\begin{array}{l}\beta=\sqrt{21 / 32 \pi} \\
\text { err }=1 \times 10^{-2}\end{array}$ \\
\hline $7 H_{g}(4)$ & $\begin{array}{l}Y_{40} ; \sqrt{7 / 15}\left(\sqrt{8 / 7} Y_{4, \pm 1} \pm Y_{4, \mp 4}\right) \\
\sqrt{1 / 15}\left(Y_{4, \pm 2} \pm \sqrt{14} Y_{4, \mp 3}\right)\end{array}$ & $\begin{array}{l}\left(35 z^{4}-30 z^{2}+3\right) \sqrt{3} \\
8 x z\left(7 z^{2}-3\right)-7\left(x^{4}+y^{4}-6 x^{2} y^{2}\right) \\
8 y z\left(7 z^{2}-3\right)+28 x y\left(x^{2}-y^{2}\right) \\
2\left(7 z^{2}-1\right)\left(x^{2}-y^{2}\right)+28 x z\left(x^{2}-3 y^{2}\right) \\
4 x y\left(7 z^{2}-1\right)-28 y z\left(3 x^{2}-y^{2}\right)\end{array}$ & $\begin{array}{l}\beta=\beta_{0} / \sqrt{56} \\
\text { err }=2 \times 10^{-2} \\
\beta_{0}=\sqrt{21 / 32 \pi}\end{array}$ \\
\hline $8 H_{u}(5)$ & $\begin{array}{l}\left(m_{2}=0\right)\left(Y_{5,5}+Y_{5,-5}\right) / \sqrt{2} \\
\left(m_{2}=\mp 1\right)+\sqrt{3 / 10} Y_{5, \pm 4} \pm \sqrt{7 / 10} Y_{5, \mp 1} \\
\left(m_{2}=\mp 2\right) \mp \sqrt{3 / 5} Y_{5, \pm 3}+\sqrt{2 / 5} Y_{5, \mp 2}\end{array}$ & $\begin{array}{l}y\left(y^{4}-10 x^{2} y^{2}+5 x^{4}\right) \sqrt{3} \\
3 z\left(x^{4}+y^{4}-6 x^{2} y^{2}\right)+x P_{51} \\
12 x y z\left(x^{2}-y^{2}\right)-y P_{51} \\
P_{51}=\left(21 z^{4}-14 z^{2}+1\right) \\
x\left(x^{2}-3 y^{2}\right) P_{53}+4\left(x^{2}-y^{2}\right) P_{52} \\
y\left(3 x^{2}-y^{2}\right) P_{53}-8 x y P_{52} \\
P_{52}=3 z^{3}-z, P_{53}=9 z^{2}-1\end{array}$ & $\begin{array}{l}\beta=\frac{\sqrt{21}}{8} \sqrt{\frac{11}{8 \pi}} \\
\text { err }=10^{-3}\end{array}$ \\
\hline $9 T_{1 u}(5)$ & $\begin{array}{l}\sqrt{7 / 25}\left(-Y_{55}+Y_{5,-5}\right) / \sqrt{2}+\sqrt{18 / 25} Y_{50} \\
\sqrt{7 / 10}\left(Y_{5, \pm 4} \mp \sqrt{3 / 7} Y_{5, \mp 1}\right)\end{array}$ & $\begin{array}{l}{\left[7 x\left(x^{4}-10 x^{2} y^{2}+5 y^{4}\right)+2 z\left(63 z^{4}-\right.\right.} \\
\left.\left.70 z^{2}+15\right)\right] / 5 ; 7 z\left(x^{4}+y^{4}-6 x^{2} y^{2}\right)-x P_{51}, \\
28 x y z\left(x^{2}-y^{2}\right)+y P_{51} \\
P_{51}=\left(21 z^{4}-14 z^{2}+1\right)\end{array}$ & $\begin{array}{l}\beta=\frac{3}{8} \sqrt{\frac{11}{8 \pi}}, \\
\text { err }=10^{-2}\end{array}$ \\
\hline $10 T_{1 g}(6)$ & $\begin{array}{l}\left(m_{1}=0\right)\left(Y_{6,5}+Y_{6,-5}\right) / \sqrt{2} \\
\left(m_{1}= \pm 1\right)-\frac{\sqrt{66}}{10} Y_{6, \pm 1} \mp \frac{\sqrt{22}}{10} Y_{6, \mp 4} \pm \frac{\sqrt{3}}{5} Y_{6, \pm 6}\end{array}$ & $\begin{array}{l}P_{65} \sin 5 \varphi=10 z y\left(y^{4}-10 x^{2} y^{2}+5 x^{4}\right) ; \\
-x P_{61}+\left(x^{4}+y^{4}-6 x^{2} y^{2}\right) P_{64}- \\
\left\{x^{2}\left(x^{2}-3 y^{2}\right)^{2}-y^{2}\left(3 x^{2}-y^{2}\right)^{2}\right\}, \\
y P_{61}+4 x y\left(x^{2}-y^{2}\right) P_{64}+2 x y\left(3 x^{2}-y^{2}\right) \\
\times\left(x^{2}-3 y^{2}\right) ; P_{61}=2\left(33 z^{5}-30 z^{3}+5 z\right), \\
P_{64}=11 z^{2}-1, P_{65}=10 z\end{array}$ & $\begin{array}{l}\beta=\frac{3}{80} \sqrt{\frac{13 \times 77}{8 \pi}} \\
\text { err }=10^{-10}\end{array}$ \\
\hline
\end{tabular}

произведение раскладывается в прямую сумму представлений: $H_{u} \otimes T_{1 g}=T_{1 u} \oplus T_{2 u} \oplus G_{u} \oplus H_{u}$. Для сравнения $H_{u} \otimes T_{1 u}=T_{1 g} \oplus T_{2 g} \oplus G_{g} \oplus H_{g}$. Представление $T_{1 u}$ в разложении $H_{u} \otimes T_{1 g}$ соответствует по симметрии орбитальному моменту $L=1$ и обеспечивает дипольный переход из основного состояния $\left(H_{u}\right)^{10}$ с симметрией $A_{g}$ в возбужденное состояние $\left(H_{u}\right)^{9}\left(T_{1 g}\right)^{1}$. Для возбужден- ного состояния $\left(H_{u}\right)^{9}\left(T_{1 u}\right)^{1}$, наоборот, доступен квадрупольный переход из основного состояния $\left(H_{u}\right)^{10}$ посредством представления $H_{g}(L=2)$ в разложении. Уровни энергии, соответствующие представлению $10 T_{1 g}$ (6), могут быть заселены и освобождены излучательным образом посредством дипольного перехода из основного состояния и назад в основное состояние. В случае 
Таблица 5. Интеграл в форме Гаунта для наилучших приближений неприводимых представлений $T_{1 g}, H_{u}$ посредством сферических функций и сопряженной функции к функции $Y_{1 m}$ (только ненулевые комбинации)

\begin{tabular}{|c|c|c|c|c|}
\hline \multirow[t]{2}{*}{ № } & \multicolumn{3}{|c|}{ Представление } & \multirow{2}{*}{$\mid \begin{array}{c}\text { Интеграл } \times \sqrt{4 \pi / 3} \\
\beta^{-1}=\sqrt{\left(2 l_{1}+1\right)}\end{array}$} \\
\hline & $T_{1 g}, l_{1}=6, m_{1}$ & $H_{u}, l_{2}=5, m_{2}$ & $T_{1 u}, l=1, m$ & \\
\hline 1 & 0 & 0 & 0 & $-\beta$ \\
\hline 2,3 & \pm 1 & 0 & \pm 1 & $-\beta / 2$ \\
\hline 4,5 & 0 & $\mp 1$ & $\mp 1$ & $\sqrt{3} \beta / 2$ \\
\hline 6,7 & \pm 1 & $\mp 1$ & 0 & $\sqrt{3} \beta / 2$ \\
\hline 8,9 & \pm 1 & $\mp 2$ & $\mp 1$ & $-\sqrt{3 / 2} \beta$ \\
\hline
\end{tabular}

возбуждения электронным ударом доступны сценарии с изменением орбитального момента на две единицы.

Основа всех квантовых расчетов - интеграл Гаунта и коэффициенты Вигнера [18]:

$$
\begin{aligned}
I & =\int_{0}^{2 \pi} d \varphi \int_{0}^{\pi} \sin \vartheta d \vartheta Y_{l, m}^{*}(\vartheta \varphi) Y_{l 1, m 1}(\vartheta \varphi) Y_{l 2, m 2}(\vartheta \varphi) \\
& =\left[\frac{\left(2 l_{1}+1\right)\left(2 l_{2}+1\right)}{4 \pi(2 l+1)}\right]^{1 / 2} C_{000}^{l_{1} l_{2} l} C_{m_{1} m_{2} m}^{l_{1} l_{2} l} .
\end{aligned}
$$

Пусть $l_{1}=l_{2}+l, l=1$, тогда коэффициенты Вигнера вычисляются по формуле

$$
\begin{aligned}
C_{m_{1} m_{2} m}^{l_{1} l_{2} l} & =\delta\left(m_{1}+m_{2}, m\right)\left[\frac{\left(2 l_{2}\right) !(2 l+1) !}{\left(2 l_{1}+1\right) !}\right]^{1 / 2} \\
& \times \frac{(-1)^{l_{2}+m_{2}} \sqrt{\left(l_{1}+m_{1}\right) !\left(l_{1}-m_{1}\right) !}}{\sqrt{(l+m) !(l-m) !} \sqrt{\left(l_{2}+m_{2}\right) !\left(l_{2}-m_{2}\right) !}} .
\end{aligned}
$$

Поэтому интеграл в форме Гаунта

$$
I_{g}=\int_{0}^{2 \pi} d \varphi \int_{0}^{\pi} \sin \vartheta d \vartheta \Psi\left(T_{1 g}, m_{1}\right) \Psi\left(T_{H u}, m_{2}\right) Y_{1 m}^{*}
$$

описывает свойства дипольных переходов с подуровня на подуровень в зависимости от комбинации квантовых чисел $\left(m_{1}, m_{2}, m\right)$; все нетривиальные комбинации сведены в табл. 5 .

Неупругие столкновения быстрых электронов с атомами и молекулами могут быть рассмотрены с помощью борновского приближения [17] (§148). Условие применимости борновского приближения требует, чтобы скорость падающего электрона была велика по сравнению со скоростями атомных электронов. Что касается потери энергии при столкновении, то она может быть любой. Если электрон теряет значительную часть своей энергии, то это приводит к ионизации молекулы, причем энергия передается одному из электронов. Неупругое столкновение сопровождается изменением внутреннего состояния системы. Квантовая система может перейти из основного состояния в возбужденное состояние дискретного либо непрерывного спектра. Вероятность перехода на уровень $\mid n>$ с энергией $E_{n}$ из основного состояния с уровнем энергии $E_{0}$ задается следующей формулой (где импульс $\mathbf{p}=\hbar \mathbf{k}$ связан с волновым вектором посредством постоянной Планка):

$$
d w_{n}=\frac{2 \pi}{\hbar}\left|\left\langle n, \mathbf{p}^{\prime}|U| 0, \mathbf{p}\right\rangle\right|^{2} \delta\left(\frac{p^{\prime 2}-p^{2}}{2 m}+E_{n}-E_{0}\right) \frac{d^{3} p^{\prime}}{(2 \pi \hbar)^{3}} .
$$

Здесь матричный элемент берется от оператора энергии взаимодействия падающего электрона с каждым атомом фуллерена:

$$
U=\frac{Z e^{2}}{r}-\sum_{a=1}^{Z} \frac{e^{2}}{\left|r-r_{a}\right|}
$$

Далее для расстояний и энергии удобно использовать атомные единицы. То есть координаты вычисляются в единицах $a_{0}=\hbar^{2} / m e^{2}$, где $a_{0}-$ боровский радиус, а энергия - в ридбергах $R_{y}=e^{2} / 2 a_{0}$. Подставим волновые функции электронов - рассеянного $\psi_{\mathbf{p}^{\prime}}=\exp \left(i \mathbf{k}^{\prime} \mathbf{r}\right)$ и исходного $\psi_{\mathbf{p}}=\sqrt{1 / k} \exp (i \mathbf{k r})$. Отметим, что начальная волновая функция электрона нормирована на единичную плотность потока. При такой нормировке $d w_{n}$ есть площадь дифференциального сечения рассеяния $d \sigma$. Если конечное состояние квантовой системы $\psi_{n}$ относится к дискретному спектру, то, как и начальное состояние $\psi_{0}$, оно нормировано на единицу. Получающиеся сечения определяют вероятность столкновения с переходом на данный уровень $n$. Интегрирование по абсолютной величине импульса $p^{\prime}$ дает

$$
d w_{n}=\left|\left\langle n, \mathbf{p}^{\prime}|U| 0, \mathbf{p}\right\rangle\right|^{2} \frac{m p^{\prime}}{4 \pi^{2} \hbar^{4}} d o^{\prime} .
$$

В силу ортогональности волновых функций связанного электрона $\psi_{n}$ и $\psi_{0}$ взаимодействие с ядром углерода исчезает и остается только взаимодействие с электронами (сохраняем в рассмотрении только один $\pi$-электрон на атом), которое спадает как $q^{-4}$. Ввиду

$$
\int \frac{e^{-i \mathbf{q r}}}{\left|r-r_{a}\right|} d V=\frac{4 \pi}{q^{2}} e^{-i \mathbf{q r}_{a}}
$$

получим

$$
d \sigma_{n}=\left|\left\langle n\left|e^{-i \mathbf{q r} r_{a}}\right| 0\right\rangle\right|^{2} \frac{4}{a_{0}^{2} q^{4}} \frac{k^{\prime}}{k} d o^{\prime}
$$

Здесь

$$
\left\langle n\left|e^{-i \mathbf{q} \mathbf{r}_{a}}\right| 0\right\rangle=\int \psi_{n}^{*} e^{-i \mathbf{q} \mathbf{r}_{a}} \psi_{0} d \tau
$$

- матричный элемент, в котором интегрирование идет по волновым функциям неприводимых представлений. Суммирование идет по числу электронов системы в данном представлении, которые испытывают переход. В случае одноквантовых процессов возбуждается 
только один электрон системы, а остальные остаются без изменения. Поскольку $\mathbf{q}=\mathbf{k}^{\prime}-\mathbf{k}$, тогда для его абсолютной величины можно записать формулу $q^{2}=k^{2}+k^{\prime 2}-2 k k^{\prime} \cos \vartheta$, которая определяется импульсами падения-рассеяния и углом рассеяния $\vartheta$. Кроме того, $q d q=k k^{\prime} \sin \vartheta d \vartheta=\frac{k k^{\prime}}{2 \pi} d o$, тогда формула сечения возбуждения имеет вид

$$
d \sigma_{n}=\left|\left\langle n\left|e^{-i \mathbf{q r}_{a}}\right| 0\right\rangle\right|^{2} \frac{8 \pi d q}{\left(a_{0} k\right)^{2} q^{3}} .
$$

Дальнейшее упрощение можно получить, ограничив рассмотрение дипольным приближением, разложив экспоненту до линейных членов. Направим ось $\xi$ вдоль вектора q, тогда формула (11) приобретает окончательный вид (матричный элемент с точностью до заряда электрона есть дипольный момент, $\xi_{z}-$ безразмерная координата электрона, совершающего переход из состояния, например, $H_{u}\left(m_{2}=0\right)$ в состояние $T_{1 g}\left(m_{1}=0\right)$ :

$$
d \sigma_{T 1 g, 0}=\left.\left|\left\langle T_{1 g}, 0\left|\left(\xi_{z} \mathbf{n}_{z}, \mathbf{n}_{q}\right)\right| H_{u}, 0\right)\right\rangle\right|^{2} \frac{8 \pi d q}{k^{2} q} .
$$

Введем угол между вектором $\mathbf{q}$ и осью $z$ молекулы фуллерена как угол $\chi$. Чтобы превратить матричный элемент в интеграл в форме Гаунта, надо преобразовать координату $z$ в $Y_{10}$ с помощью радикала $\sqrt{3 / 4 \pi}$. Тогда в выражении (12) матричный элемент по модулю в квадрате есть

$$
\left.\left|\left\langle T_{1 g}, 0\left|\xi_{z} \cos \chi\right| H_{u}, 0\right)\right\rangle\right|^{2}=\mu_{00}^{2}\left\langle\cos ^{2} \chi\right\rangle
$$

и

$$
\begin{aligned}
\mu_{00}^{2} & =\left|\left\langle R_{1,5}\left|r / a_{0}\right| R_{1,6}\right\rangle\right|^{2}\left(2 l_{1}+1\right)^{-1} \\
& \approx\left(R_{f} / a_{0}\right)^{2}\left(2 l_{1}+1\right)^{-1} .
\end{aligned}
$$

Здесь $\left\langle R_{n 5}\left|r / a_{0}\right| R_{n 6}\right\rangle$ - матричный элемент для радиальной части нормированных волновых функций $\left\langle R_{n l} \mid R_{n l}\right\rangle=1$ и $R_{f} / a_{0}$ - радиус молекулы фуллерена в атомных единицах (в единицах $a_{0}$ ). Численный расчет радиального матричного элемента дает значение радиуса фуллерена в атомных единицах с точностью до единиц процента. Потенциальная яма в фуллерене - это сферическая яма на расстоянии $R_{f}$ от центра. Поэтому матричный элемент на сферической оболочке близок к значению интеграла с $r=R_{f}$ в точке дна ямы.

Потенциал в атомных единицах $\left(2 m U / \hbar^{2}\right)=2 u$, $\eta=R_{f} / a_{0}$ равен:

$$
\begin{aligned}
& 2 u\left(x \equiv r / a_{0}\right)= \\
& -\left\{\frac{\eta}{0.61^{2}+\left|x^{2}-\eta^{2}\right|}+\frac{1}{\eta}-\frac{(x-\eta)^{2}}{\eta x^{2}} \theta(x-\eta)\right\} .
\end{aligned}
$$

Для усреднения по углам $\chi$ расположим вспомогательную систему координат в плоскости треугольника, натянутого на векторы $-\mathbf{k}, \mathbf{k}^{\prime}$, совместив начало второго вектора с концом первого. Угол $\vartheta$ задает длину вектора $\mathbf{q}=\mathbf{k}^{\prime}-\mathbf{k}$. Направим ось $z^{\prime}$ параллельно оси вектора падения и ось $x^{\prime}$ в плоскости треугольника и перпендикулярно полярной оси. Угол $\chi$ запишем через скалярное произведение единичного вектора вдоль оси $z$ и оси $q$ :

$$
\cos \chi=\cos \vartheta_{q} \cos \vartheta_{z}+\sin \vartheta_{q} \sin \vartheta_{z} \cos \varphi_{z}
$$

Тогда усреднение $\left\langle\cos ^{2} \chi\right\rangle_{z}$ по всем направлениям вектора $\mathbf{z}$ приводит к 1/3. В итоге (12) преобразуется к виду (так как из закона сохранения энергии квадраты волновых векторов различаются на константу $\left.\left(k^{2}-k^{\prime 2}\right)=\frac{\Delta E}{a_{0}^{2} R_{y}}=\mu^{2}\right)$

$$
\sigma_{T 1 g, 0}=\frac{1}{3} \frac{\mu_{00}^{2}}{(k)^{2}} \int \frac{8 \pi d q}{q}=\frac{1}{3} \mu_{00}^{2} \frac{8 \pi}{k^{2}} \ln \frac{k+\sqrt{k^{2}-\mu^{2}}}{k-\sqrt{k^{2}-\mu^{2}}}
$$

Размерность $[k]=\left[a_{0}\right]^{-1}-$ размерность волнового вектора. Для переходов из состояния $H_{u}\left(m_{2}=0\right)$ в состояние $T_{1 g}\left(m_{1}= \pm 1\right)$ матричный элемент

$$
\left\langle\left|\frac{\left(\mathbf{n}_{x} \xi_{x}+i \mathbf{n}_{y} \xi_{y}, \mathbf{n}_{q}\right)}{\sqrt{2}}\right|^{2}\right\rangle=\frac{\mu_{ \pm 1,0}^{2}}{3}=\frac{\mu_{00}^{2}}{12}
$$

а множитель перехода к $Y_{11}$ равен $\sqrt{3 / 8 \pi}$. Система уровней $H_{u}\left(l_{2}=5\right)$ имеет пятикратное вырождение. Верхний уровень $T_{1 g}\left(l_{1}=6\right)$ трехкратно вырожден. Таким образом, при возбуждении на данном переходе можно реализовать равновероятно один из 15 вариантов. Мы имеем дело с условной вероятностью. Усреднение проведем вначале по верхнему уровню, затем по нижнему. Усредненное сечение - половина $\sigma_{T 1 g, 0}$ (здесь $\varepsilon=E / R_{y}=k^{2} a_{0}^{2}$ - энергия электрона и $\Delta \varepsilon=\Delta E / R_{y}$ разность энергий двух уровней в ридбергах):

$$
\sigma_{H u 0 \rightarrow T 1 g}=\frac{(4 \pi / 3) R_{f}^{2}}{\left(2 l_{1}+1\right)} \frac{1}{\varepsilon} \ln \frac{(\sqrt{\varepsilon}+\sqrt{\varepsilon-\Delta \varepsilon})^{2}}{\Delta \varepsilon} .
$$

Итак, сечение возбуждения с любого уровня системы $H_{u}$ на допустимые по правилу отбора подуровни $T_{1 g}$ определяется выражением (15). Разложение экспоненты до линейных членов в формуле (11) при выводе (12) опирается на ряд Тейлора, однако более строгое разложение экспоненты опирается на формулу

$$
e^{i \mathbf{q r}}=\sum_{l=0}^{\infty}(2 l+1) i^{l} j_{l}(q r) P_{l}\left(\mathbf{n}_{\mathbf{q}} \mathbf{n}_{\mathbf{r}}\right)
$$

в которой при разложении до членов первого порядка по полиномам Лежандра $-P_{1}\left(\mathbf{n}_{\mathbf{q}} \mathbf{n}_{\mathbf{r}}\right)-$ радиальная зависимость выражается через сферические функции Бесселя $3 j_{1}(q r)$. Тогда для радиального матричного элемента получим несколько другой результат ввиду нарушения условия применимости разложения в ряд Тейлора $q r \ll 1$ в нашем случае. Результаты расчета по обоим вариантам приведены на рис. 3 , где видно, что результаты отличаются примерно в три раза. 


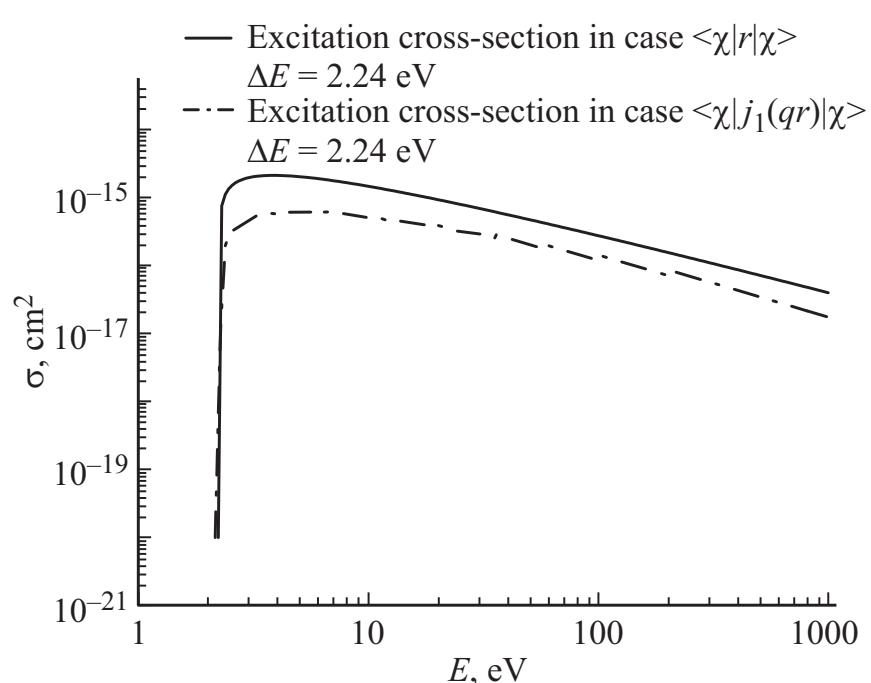

Рис. 3. Сечение возбуждения трехкратно вырожденного уровня с симметрией $T_{1 g}$ в дипольном приближении (ряд Тейлора и ряд по полиномам Лежандра) электронами с энергией до $20 \mathrm{eV}$. Рассматриваются переходы с пятикратно вырожденного уровня $H_{u}$. Этот уровень полностью заселен (система фуллерена нейтральна). Выше лежит незаселенный уровень с симметрией $T_{1 u}$, переходы на который оптически запрещены, а за ним незаселенный $T_{1 g}$. Порог возбуждения $2.24 \mathrm{eV}$. Максимум приходится на $3.9 \mathrm{eV}$. Ввиду нарушения условия $q r \ll 1$, второй вариант более строг.

Вероятность спонтанного излучения отдельного уровня на свободный с меньшей энергией дается в дипольном приближении формулой [19]:

$$
d W_{a b}=\frac{\alpha^{4} c(\Delta \varepsilon)^{3}}{16 \pi a_{0}}\left|\left(\mathbf{e}_{k p},\left\langle a\left|\mathbf{r} / a_{0}\right| b\right\rangle\right)\right|^{2} d o .
$$

Здесь матричный элемент вычисляется в безразмерных атомных единицах, энергия перехода берется в ридбергах, $\alpha=e^{2} / \hbar c$ - постоянная тонкой структуры и $c$ - скорость света. Направим ось $z$ по направлению распространения оптической волны. Оптическое поле поперечно, выберем векторы поляризаций так, чтобы $\left(\mathbf{e}_{k 1}, \mathbf{n}_{\mathbf{r}}\right)=\sin \vartheta,\left(\mathbf{e}_{k 2}, \mathbf{n}_{\mathbf{r}}\right)=0$. После усреднения по телесному углу, так как

$$
\int \sum_{p=1}^{2}\left(\mathbf{e}_{k p}, \mathbf{n}_{\mathbf{r}}\right) d o=\int_{0}^{2 \pi} d \varphi \int_{0}^{\pi} \sin \vartheta d \vartheta \sin ^{2} \vartheta=\frac{8 \pi}{3},
$$

получим:

$$
W_{a b}=\frac{\alpha^{4} c(\Delta \varepsilon)^{3}}{6 a_{0}}\left|\left\langle a\left|\mathbf{r} / a_{0}\right| b\right\rangle\right|^{2} .
$$

Тогда излучение с подуровня $T_{1 g}\left(m_{1}=0\right)$ на подуровень $H_{u}\left(m_{2}=0\right)$ задается выражением

$$
W_{a b}=\frac{\alpha^{4} c(\Delta \varepsilon)^{3}}{6 a_{0}} \frac{\left(R_{f} / a_{0}\right)^{2}}{2 l_{1}+1} .
$$

Для излучения с любого конкретного подуровня $T_{1 g}$ на свободный оптически разрешенный подуровень $H_{u}$ после усреднения по всем допустимым вариантам получим множитель (5/6) перед выражением (18), и обратное время жизни перехода равно

$$
\tau_{a b}^{-1}=\frac{\alpha^{4} c(\Delta \varepsilon)^{3}}{36 a_{0}} \frac{5 R_{f}^{2} / a_{0}^{2}}{2 l_{1}+1}
$$

Здесь $R_{f} / a_{0}-$ радиус молекулы фуллерена в атомных единицах и $\Delta \varepsilon=\Delta E / R_{y}-$ разность энергий двух уровней фуллерена в ридбергах.

\section{5. Заключение}

Рассмотренная модель молекулярных орбиталей позволяет найти спектр электронных уровней молекулы фуллерена $\mathrm{C}_{60}$ с помощью теории неприводимых представлений группы усеченного икосаэдра. Для заданной геометрии фуллерена вычислен параметр $\alpha$, минимизирующий погрешность разложения по сферическим функциям. Найдено 16 уровней энергии, степень вырождения уровня (без учета направления спина) меняется в диапазоне от 1 до 5. Построена таблица угловых волновых функций для первых 10 нижних уровней. Первые 8 из них полностью заполнены (30 различных волновых функций). Еще 2 представления описывают незаполненные трехкратно вырожденные уровни. Найдено разложение представлений $7 H_{g}(4)$ и $9 T_{1 u}(5)$ по сферическим функциям. Из-за правил симметрии оптические переходы между верхним заполненным $\left(H_{u}\right)$ и нижним незаполненным $\left(T_{1 u}\right)$ уровнями в дипольном представлении невозможны. Зато на следующий свободный уровень оптические переходы разрешены. Используя значение радиуса молекулы фуллерена и разность значений энергий между верхним и нижним уровнями, можно получить значения собственного времени жизни возбужденного состояния и сечение возбуждения в рамках развитого выше подхода разложения неприводимых представлений по сферическим функциям. Это позволяет вычислить матричные элементы переходов с помощью коэффициентов Вигнера и коэффициентов разложения неприводимых представлений по сферическим функциям. В качестве иллюстрации рассмотрен первый оптический переход. Кратность вырождения верхнего состояния 3 (свойства любого из подуровней нижнего состояния совпадают). Радиус фуллерена $R_{f}=3.508 \AA$, радиус Бора $a_{0}=\hbar^{2} / m e^{2}=0.53 \AA$, следовательно, $R_{f} / a_{0}=6.62$. Разность энергий двух рассматриваемых вырожденных уровней $\Delta \varepsilon=\Delta E / R_{y}=0.165$. Орбитальный момент в возбужденном состоянии равен 6. Тогда время жизни перехода $2.24 \mathrm{eV}$ составляет $\tau_{2.24}=0.27 \mathrm{~ns}$. Сечение возбуждения электрона с энергией $\varepsilon_{e v}$, согласно формуле (15) и графику сечения возбуждения на рис. 3, имеет максимум на $3.9 \mathrm{eV}$, который достигает значения $2.15 E-15 \mathrm{~cm}^{2}$. С использованием более точного разложения по сферическим функциям Бесселя та же 
величина равна $6 . E-16 \mathrm{~cm}^{2}$. При расчете радиального матричного элемента был использован код быстрого преобразования Ханкеля полуцелого порядка для линейной координатной сетки. Центральный потенциал (13), подставленный в уравнение Шредингера, позволяет найти уровни энергии в одноэлектронном приближении, результаты даны в правой колонке табл. 2. Сравнение с методом молекулярных орбиталей показывает, что происходит сильное расщепление на ряд подуровней, различных по энергии, но с одним и тем же квантовым числом $L$. Многочастичное описание выявляет понижение симметрии $\mathrm{SO}(3)$ до симметрии икосаэдра, в котором частично снимается вырождение по проекции момента.

Угловые части матричных элементов определяются неприводимыми представлениями обоих уровней, между которыми осуществляется переход, и могут быть вычислены с использованием разложения функций на сферические гармоники с помощью табл. 4 и через коэффициенты Вигнера. Полученные матричные элементы позволяют найти сечения возбуждения фуллерена и собственные времена жизни возбужденных состояний. Однако картина будет не полна, если не учитывать следующие три момента. Во-первых, возбуждения могут происходить не только дипольным образом, но и квадрупольно с переворотом спина электрона. В этом случае вместо таблицы рассмотренных неприводимых представлений икосаэдра применяется таблица характеров двойной группы икосаэдра, работающей как для целых, так и полуцелых значений вектора суммарного момента $J=L+S$. Во-вторых, в системе фуллерена очень активно электрон-фононное взаимодействие, связанное с эффектом Яна-Теллера. Из-за эффекта происходит спонтанное нарушение исходной симметрии икосаэдра с возбуждением фононных колебаний. При понижении симметрии снимается вырождение, и система вырожденных уровней для исходных неприводимых представлений вновь расщепляется по энергии. С учетом расщепления снимаются некоторые запреты на переходы между подсистемами уровней. В-третьих, наряду с оптическим каналами релаксации возбуждения появляются также безызлучательные переходы, снимающие возбуждение электронных уровней. Прямой эксперимент на отрицательно заряженном ионе фуллерена по определению скорости безызлучательной релаксации был выполнен в работе [20]. С помощью коротких фемтосекундных импульсов реализована двухлучевая фотоэлектронная спектроскопия первого возбужденного уровня иона фуллерена и измерено его время безызлучательной релаксации. Полученное время жизни уровня имеет порядок двух пикосекунд, что почти на три порядка меньше оптических времен жизни. Для описания механизма релаксации с учетом сильной связи электронных состояний с фононами решетки фуллерена подходит модель БорнаОппенгеймера, предложенная в работе [21]. Уширение линии (а значит, и обратное время жизни) пропорционально квадрату матричного элемента перехода между изучаемыми состояниями и обратно пропорционально энергии фонона. Вероятность заселения возбужденного уровня спадает экспоненциально во времени сразу после заселения уровня. Быстрый распад возбужденного состояния делает сложным наблюдение флуоресценции в оптическом диапазоне, однако в микроволновом диапазоне существует тепловое излучение, индуцированное фононным спектром [22]. Константы электрон-фононной связи и энергии фононов в молекуле фуллерена изучались авторами [23].

Таким образом, молекула фуллерена обладает свойствами симметрии икосаэдра, что задает интересные особенности ее спектра. Однако за счет электронфононной связи симметрия может спонтанно нарушаться с перекачкой энергии из электронной части системы в фононную. Безызлучательная релаксация очень быстрая (здесь все происходит на тех же временах, как в полупроводниках). Чтобы увидеть электронный спектр излучения молекулы, нужны внутрирезонаторные схемы, так как вероятность индуцированных оптических переходов пропорциональна числу фотонов. Электронный спектр будет изрезан фононными обертонами.

\section{Благодарности}

Работа выполнена при финансовой поддержке проекта Росатома № 313/1731-Д от 19.09.2019. Работа посвящается памяти выдающегося ученого А.Н. Старостина, посвятившего себя исследованию квантовых многочастичных систем. Особенную признательность за поддержку при выполнении работ выражаю И.В. Кочетову, Н.Н. Дятко и также руководителю проекта А.А. Якушкинy.

\section{Финансирование работы}

Работа выполнена при финансовой поддержке проекта Росатома № 313/1731-Д от 19.09.2019.

\section{Конфликт интересов}

Автор заявляет, что у него нет конфликта интересов.

\section{Список литературы}

[1] Клейн Ф. Лекции об икосаэдре и решении уравнений пятой степени. М.: Наука, 1989.

[2] Dresselhaus M.S., Dresselhaus G., Eklund P.C. Science of Fullerenes and Carbon Nanotubes. Academic Press, 1996.

[3] Haddon R.C., Brus L.E., Raghavachari Krishnan // Chem. Phys. Lett. 1986. V. 125. N 5-6. P. 459 -464.

[4] Deng Y, Yang C.N. // Phys. Lett. 1992. V. 170. P. 116-126.

[5] Эллиот Джс, Доббер П. Симметрия в физике, в двух томах. М.: Мир, 1983.

[6] Shi-Hai Dong, Xi-Wen Hou, Mi Xie, Zhong-Qi Ma. // International J. of Theoret. Phys. / 1998. V. 37. N 8. P. 2135 2152. 
[7] Atkins P.W, Friedman R.S. Molecular Quantum Mechanics. NY.: Oxford University Press, 3d-edition, 1996.

[8] Fowler P.W., Woolrich J. // Chem. Phys. Lett. 1986. V. 127. N 1. P. $78-83$.

[9] Chancey C.C., O'Brien M.C.M. The Jahn-Teller Effect in $\mathrm{C}_{60}$ and Other Icosahedral Complexes. Princeton University Press, 1997.

[10] Силантьев А.В. // Физика металлов и металловедение. 2017. V. 118. № 1. P. 3-11.

[11] Арутюнян Р.В., Васильев А.Д. и др. // Отчет о НИР „Расчетно-теоретические исследования характеристик электронных уровней в заряженных фуллеренах $\mathrm{C}_{60}{ }^{*}$. АНО ЦАБ ИБРАЭ РАН, эТап 1. 2019.

[12] Hawkins J.M., Meyer A., Lewis T.A., Loren S., Hollander F.J. // Science. 1991. V. 252. P. 312-313.

[13] Трошин П.А., Любовская Р.Н. // Успехи химии. 2008. V. 77. № 4. P. 323-369.

[14] Гантмахер Ф.Р. Теория матриц. М.: Наука, 1988.

[15] Qihuang Gong, Yuxing Sun, Ziwen Huang, Xihuang Zhu, Gu Z.N., Di Qiang // J. Phys. B: At. Mol. Opt. Phys. 1994. V. 27. P. L199-L201.

[16] Christophe Bulliard, Michael Allan, Sydney Leach // Chem. Phys. Lett. 1993. V. 209. N 5-6. P. 434-438.

[17] Ландау Д.Д., Лифиии, Е.М. Теоретическая физика, том 3. Квантовая механика. Нерелятивисткая теория. М.: Наука, 1989.

[18] Биденхарн Л., Лаук Джс. Угловой момент в квантовой физике. Теория и приложения в 2-х томах. Том 1. М.: Мир, 1984.

[19] Собельман И.И. Введение в теорию атомных спектров. М.: Наука, 1977.

[20] Oli T. Ehrler, Ji Ping Yang, Christof Hättig, AndreasN. Unterreiner, Horst Hippler, Manfred M. Kappes // J. Chem. Phys. 2006. V. 125. P. 074312.

[21] Mordechai Bixon, Joshua Jortner // J. Chem. Phys. 1968. V. 48. P. 715.

[22] Востриков А.А., Дубов Д.Ю., Агарков А.А. // Письма в ЖЭТФ. 1996. V. 63. № 12. Р. 915-919.

[23] Antropov V.P., Gunnarsson O., Liechtenstein A.I. // Phys. Rev. B. 1993. T. 48. N 10. P. 7651-7664. 